\title{
Microscopic treatment of fluctuations in finite quantum systems
}

\author{
R. Rossignoli ${ }^{1,2}$, N. Canosa ${ }^{2}$, J.L. Egido ${ }^{1}$ \\ ${ }^{1}$ Departamento de Física Teórica C-XI, Universidad Autónoma de Madrid, E-28049 Madrid, Spain \\ 2 Departamento de Física, Universidad Nacional de La Plata, C.C.67, 1900 La Plata, Argentina
}

\begin{abstract}
We describe a fully microscopic treatment of fluctuations in correlated finite systems at finite temperature, based on the static path approximation (SPA) to the partition function, which incorporates the large amplitude statistical fluctuations around mean field, and the ensuing SPA+RPA approach, which includes in addition the small amplitude quantal fluctuations. An application to the description of pairing, shape and orientation fluctuations in hot nuclei is then given. The treatment of constraints is also discussed.
\end{abstract}

Small correlated quantum systems exhibit important fluctuation phenomena. At finite temperature, statistical fluctuations in the relevant order parameters smooth the sharp phase transitions arising in the mean field approximation, which represent normally the behavior of the system in the limit of infinite particle number or volume. In superconducting particles, these fluctuations lead to a broadening of the superfluid to normal transition when the size of the system is sufficiently small, the relevant size parameter being the ratio $\delta$ of the average single electron level spacing to $T_{c}$, which is proportional to the inverse of the volume [1]. Fluctuation effects become important for $\delta>0.01$, in which case deviations from the sharp BCS behavior are significant. Similarly, in hot finite nuclei fluctuations in the shape deformation and gap parameters smooth out the corresponding transitions arising in the mean field [2]. The concomitant effects become manifest in several aspects of hot nuclei formed in heavy ion collisions [3], like the description of the giant dipole resonance [4] and, as recently shown, the collective decay spectra [5].

Statistical fluctuations are mostly introduced in nuclear physics by means of semi-macroscopic prescriptions $[2,3,4$, 5], in part due to the phenomenological character of the models employed. Nevertheless, a fully microscopic treatment of large amplitude statistical fluctuations can be obtained by means of the SPA, first derived in solid state physics for describing fluctuations in small superconducting particles [1], and later introduced in nuclear physics [6,7], which is exact at high temperatures. The SPA can be improved at low temperatures by the SPA+RPA $[8,9]$, which incorporates the small amplitude quantal fluctuations, and by projection onto conserved symmetries $[10,11]$. Very few realistic applications of these latter methods have so far been performed in nuclear physics. In this contribution we give a brief review of these methods, including an exact evaluation of the RPA correction factor within the SPA, together with novel results obtained for heavy nuclei.

We consider a general fermion Hamiltonian containing at most two-body terms, which can be always written as

$H=H_{0}-\frac{1}{2} \sum_{\nu} v_{\nu} Q_{\nu}^{2}$,

where $H_{0}$ and $Q_{\nu}$ are hermitian one-body (one particle or one quasiparticle) fermion operators. By means of the Hubbard-Stratonovich transformation, the grand canonical (GC) partition function can be written as the path integral [12]

$$
\begin{aligned}
Z & =\int D[x] \operatorname{Tr} \hat{T} \exp \left\{-\int_{0}^{\beta} d t H^{\prime}[x(t)]\right\}, \\
H^{\prime}(x) & \equiv H_{0}+\sum_{\nu}\left(\frac{x_{\nu}^{2}}{2\left|v_{\nu}\right|}-s_{\nu} x_{\nu} Q_{\nu}\right)-\mu N,
\end{aligned}
$$

where $\hat{T}$ denotes time ordering and $s_{\nu}=1(i)$ if $v_{\nu}>0(<0)$. With the expansion $x_{\nu}(t)=\sum_{m} x_{\nu}^{m} e^{i \omega_{m} t}, \omega_{m}=2 \pi m / \beta$, Eq. (2) becomes an integral over the coefficients $x_{\nu}^{m}$. In the SPA+RPA $[8,9]$ the integrals over the static coefficients $x_{\nu}^{0}$, which represent the time average of $x_{\nu}(t)$, are fully retained, to account for the large amplitude statistical fluctuations, whereas those over $x_{\nu}^{m \neq 0}$, representing the time dependent or quantal fluctuations, are evaluated in the saddle point approximation. The result is

$$
\begin{aligned}
Z_{\mathrm{spa}+\mathrm{rpa}} & =\int_{-\infty}^{\infty} d(x) \exp [-\beta \mathscr{F}(x)] C_{\mathrm{rpa}}(x), \\
\mathscr{F}(x) & =-\beta^{-1} \ln \operatorname{Tr} \exp \left[-\beta H^{\prime}(x)\right], \\
C_{\mathrm{rpa}}(x) & =\prod_{m=1}^{\infty} \operatorname{Det}\left[A\left(x, \omega_{m}\right)\right]^{-1}, \\
A(x, \omega) & =\delta_{\nu \nu^{\prime}}-v_{\nu} \sum_{k \neq k^{\prime}} \frac{\left\langle k\left|Q_{\nu}\right| k^{\prime}\right\rangle\left\langle k^{\prime}\left|Q_{\nu^{\prime}}\right| k\right\rangle\left(f_{k}-f_{k^{\prime}}\right),}{i \omega+\lambda_{k^{\prime}}-\lambda_{k}},
\end{aligned}
$$

where $d(x)=\prod_{\nu}\left(\beta / 2 \pi\left|v_{\nu}\right|\right)^{1 / 2} d x_{\nu}, \mathscr{F}(x)$ is an uncorrelated grand potential, $|k\rangle$ are the single particle (sp) eigen- 
states $H^{\prime}(x)|k\rangle=\lambda_{k}|k\rangle$, and $f_{k}$ the Fermi occupation probabilities. The sum over $k \neq k^{\prime}$ includes all types of fermion pairs (particle hole (ph), pp and hh). If $\left[H^{\prime}(x), N\right] \neq 0$ the sum over the enlarged quasiparticle space of doubled dimension is understood [11]. Only the static variables associated with attractive terms $v_{\nu}>0$ need to be actually considered in the integration (4). Repulsive terms do not lead to large amplitude thermal fluctuations, and the corresponding static variables can be also integrated in the saddle point method, leading to an additional determinant in (4) [13].

The product (6) can be evaluated exactly as [13]

$C_{\mathrm{rpa}}(x)=\prod_{\alpha} \frac{\omega_{\alpha}(x) \sinh \left[{ }_{2}^{1} \beta \lambda_{\alpha}(x)\right]}{\lambda_{\alpha}(x) \sinh \left[{ }_{2}^{1} \beta \omega_{\alpha}(x)\right]}$,

where $\lambda_{\alpha}(x) \equiv \lambda_{k^{\prime}}-\lambda_{k}\left(k<k^{\prime}\right)$, and $\omega_{\alpha}(x)$ are the generalized RPA frequencies defined by

$\operatorname{Det}\left[A\left(x, i \omega_{\alpha}\right)\right]=0$,

which become the ordinary thermal RPA frequencies if $x$ is a self-consistent mean field solution, i.e., $s_{\nu} x_{\nu}=v_{\nu}\left\langle Q_{\nu}\right\rangle_{x}$. If the factors $\omega_{\alpha} / \lambda_{\alpha}$ are omitted, (7) is just the ratio of the partition function of independent RPA bosons of energies $\omega_{\alpha}(x)$, to that of uncorrelated fermion pairs of energies $\lambda_{\alpha}(x)$, considered as bosons. The factors $\omega_{\alpha} / \lambda_{\alpha}$ arise due to the exclusion of the $m=0$ term in (6), i.e., due to the exact integration over the static components, and make $C_{\mathrm{rpa}}(x)>0$ and finite, even in the presence of vanishing frequencies (like Goldstone modes) and also of imaginary frequencies provided $\left|\beta \omega_{\alpha}\right|<2 \pi$. This allows to apply (7) for arbitrary $x$, as required in (4), and not just at the selfconsistent mean field, if the temperature is sufficiently high. At low $T$ and away from the stable mean field, regions where $\beta^{2} \omega_{\alpha}^{2}(x) \leq-4 \pi^{2}$ will normally arise, and (4) is no longer applicable. This indicates the failure of the gaussian approximation for the integral over $x_{\nu}^{m \neq 0}$ in these regions due to the onset of large amplitude quantal fluctuations.

The smoothing of the mean field transitions at finite temperature is already attained in the SPA, obtained by neglecting $C_{\text {rpa }}(x)$ in (4), which simply includes the statistical fluctuations around the mean field, given by the minimum of $\mathscr{T}(x)$. The SPA is valid for all $T>0$ and becomes exact at high temperatures (up to order $\beta$ ) in a finite configuration space $\left(C_{\mathrm{rpa}}(x) \rightarrow 1\right.$ for $\left.\beta \rightarrow 0\right)$. The SPA improves the standard mean field, while the SPA+RPA, when applicable, improves both SPA and mean field + RPA. The accuracy of the SPA at low $T$ will depend actually on the choice of the diagonal representation (1), which is not unique.

The present methods become particularly simple for effective models containing just separable interactions, in which the optimal diagonal form (1) is directly manifest. As an illustrative example, we consider the well known pairing plus quadrupole Hamiltonian [14],

$H=H_{0}-{ }_{2}^{1} \chi Q^{\dagger} \cdot Q-\sum_{\tau=p, n} g_{\tau} P_{\tau}^{\dagger} P_{\tau}$,

where $H_{0}$ is an effective spherical sp Hamiltonian, $Q_{\mu}$ quadrupole sp operators and $P_{\tau}^{\dagger}=\frac{1}{2} \sum_{k} c_{k \tau}^{\dagger} c_{k \tau}^{\dagger}$ monopole pairing operators. Here $\tau$ denotes isospin and $k=n l j m$ the labels of an oscillator basis, with $l,(\mathrm{jm})$ the orbital and total angular momenta. In heavy nuclei, (8) is meant as an effective Hamiltonian for valence nucleons within a restricted configuration space, apt for describing the essential features of pairing and shape deformations. More refined versions include a few higher multipoles and isovector components (see for instance [15]), although (8) is the essential part. For moderate temperatures $T<1.8 \mathrm{MeV}$, total spin $<60$, and nuclei in the range $140<A<190$, normally two oscillator shells for each component (of dimension $2 d_{\tau}$ ) are considered. The total number of many-body states in this space for a rare earth nucleus like ${ }^{164} \mathrm{Er}$ is already $2.9 \times 10^{43}$.

The SPA will involve an integral over 5 quadrupole variables $x_{\mu}$, which can be written in terms of intrinsic deformation parameters $\beta, \gamma$ and 3 orientation angles, and the pairing variables $x_{\tau}=\Delta_{\tau} e^{i \phi_{\tau}}$. For calculations in hot rotating nuclei, a cranking term $-\omega J_{z}$ is added to (8). The $U(1)$ gauge angles $\phi_{\tau}$ and one of the orientation angles can be integrated out. The final result is [6]

$$
\begin{aligned}
Z_{\mathrm{spa}}= & \mathscr{N} \int_{0}^{\infty} \beta^{4} d \beta \int_{0}^{\frac{\pi}{3}} \sin (3 \gamma) d \gamma \int d \Omega \prod_{\tau} \int_{0}^{\infty} \Delta_{\tau} d \Delta_{\tau} \\
\times & \operatorname{Tr} \exp \left\{-\left[H(\beta, \gamma, \Delta)-\sum_{\tau} \mu_{\tau} N_{\tau}-\boldsymbol{\omega} \cdot \boldsymbol{J}\right] / T\right\}, \\
H(\beta, \gamma, \Delta)= & H_{0}+\frac{\beta^{2}}{2 \chi}-\beta\left(Q_{0} \cos \gamma+\frac{Q_{2}+Q_{-2}}{\sqrt{ } 2} \sin \gamma\right) \\
& +\sum_{\tau} \frac{\Delta_{\tau}^{2}}{g_{\tau}}-\Delta_{\tau}\left(P_{\tau}^{\dagger}+P_{\tau}\right)-{ }_{2}^{1} g_{\tau}\left(N_{\tau}-d_{\tau}\right)
\end{aligned}
$$

where all operators refer now to the intrinsic frame, with $\Omega=(\theta, \phi)$ the orientation of the rotation axes $\boldsymbol{\omega}(\boldsymbol{J}$ is the total angular momentum operator). Here $\beta, \gamma, \Delta_{\nu}$ are the deformation parameters and gaps of the intrinsic potential (rather than density). Shape, pairing and orientation fluctuations are thus directly obtained, including the proper integration measure, which was not apparent in the macroscopic treatments $[2,3,4,5]$ (where the measure $\beta d \beta d \gamma d \Delta_{\tau}$ was first employed). In the SPA+RPA, a factor $C_{\mathrm{rpa}}(\beta, \gamma, \Omega, \Delta)$ is included.

So far we considered GC statistics (fixed $\mu_{\tau}$ and $\omega$ ). In a finite nucleus however, one is rather interested in statistics and fluctuations for fixed particle number and spin (or spin component). Projection in the saddle point approximation leads to the replacement [11]

$$
\begin{aligned}
& \exp \left[-\mathscr{F}\left(x, \mu_{\tau}, \omega\right) / T\right] \rightarrow \\
& \quad \exp \left[-\mathscr{F}\left(x, N_{\tau}, J\right) / T\right] /\left[(2 \pi)^{3} D\right]^{\frac{1}{2}}
\end{aligned}
$$

in the SPA integrand, where $\mathscr{F}\left(x, N_{\tau}, J\right)$ is the free energy for fixed $N_{\tau}, J$ ( $D$ is the fluctuation determinant). This implies the adjustment of $\mu_{\tau}$ and $\omega$ for each $x=(\beta, \gamma, \Omega, \Delta)$ by the corresponding constraints. The ensuing effects can be significant at low $T$ in the presence of shape coexistence, as different configurations (like prolate or oblate deformation, normal or superfluid) may have quite different values of $\mu_{\tau}, \omega$ for fixed $N_{\tau}, J$. This approximate projection is nonetheless not accurate for $T \rightarrow 0$. In the SPA, the exact projection in the form $\operatorname{Tr} P_{N J} \exp \left[-\beta H^{\prime}(x)\right]$, with $P_{N J}$ the particle number and spin projector, can be performed [10], and improves significantly the $T \rightarrow 0$ limit of expectation values. 

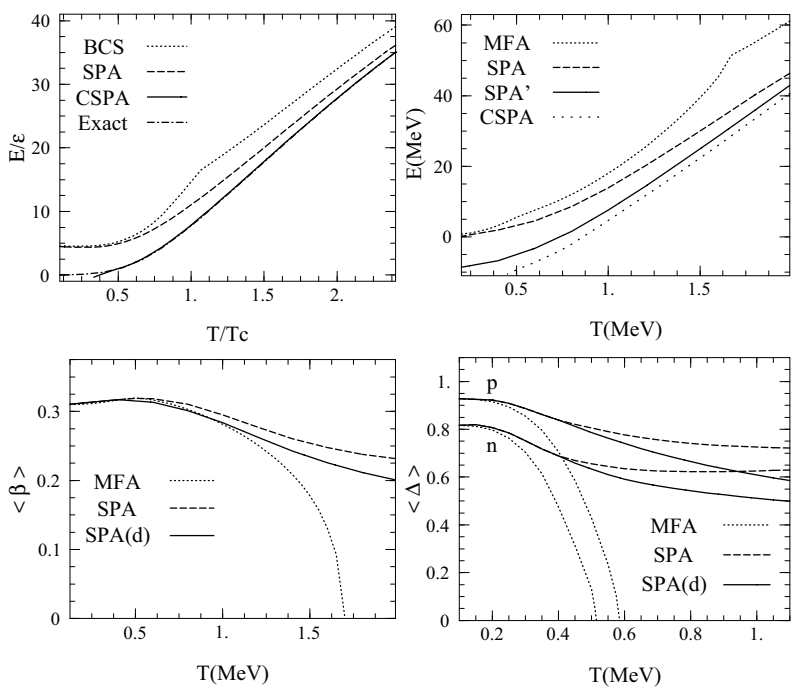

Fig. 1. Top: Left: Average thermal energy in a pairing model (see text), according to BCS, SPA, SPA+RPA (denoted as CSPA) and the exact result. Right: Average energy in the nucleus ${ }^{164} \mathrm{Er}$ for Hamiltonian (8), measured from the $T=0$ Hartree-Bogoliubov (MFA) energy. SPA' depicts the average $\langle H\rangle_{\text {spa. }}$. Bottom: Average of the quadrupole deformation parameter $\beta$ (dimensionless) and of the proton (p) and neutron (n) gaps (in MeV). Here SPA depicts the potential averages, while SPA(d) the corresponding density averages

In order to visualize the accuracy of the previous methods, we first depict in Fig. 1 the average energy in a pairing model (consisting of 20 sp levels [16], half filled, with $\left.\delta=\varepsilon / T_{c} \approx 0.7\right)$, where the partition function can be exactly calculated. The SPA energy (calculated as $-\partial \ln Z_{\text {spa }} / \partial \beta$ ) gives the correct smoothed behavior, while the SPA+RPA result is practically exact above the breakdown temperature $T \approx 0.25 T_{c}$. We show then new results for the full Hamiltonian (8) in the nucleus ${ }^{164} \mathrm{Er}$, with the parameters of ref. [14] $\left(g_{p}=27 / A, g_{n}=22 / A, \chi=70 A^{-1.4} \mathrm{MeV}\right)$. We set $\omega=0$, and employ number projection in the saddle point approach. The mean field (thermal Hartree-Bogoliubov) exhibits a deformed to spherical transition at $T \approx 1.7 \mathrm{MeV}$, and superfluid to normal transitions at $T \approx 0.6$ and $0.5 \mathrm{MeV}$ for protons and neutrons respectively, all of which appear considerably washed out in the SPA. The RPA energy correction to SPA is significant, although it consists mainly of exchange contributions. The full average $\langle H\rangle_{\text {spa }}$ containing the exchange terms [10], is closer to the SPA+RPA result. We also depict the average shape and pairing potential deformations $\langle\beta\rangle_{\mathrm{spa}},\left\langle\Delta_{\tau}\right\rangle_{\mathrm{spa}}$, and the density deformations [11], which are coincident in the MFA. Fluctuations are considerable and the deviation from the MFA behavior is significant. The influence of the integration measure is very important, particularly the factor $\beta^{4}$, which strongly unfavors spherical configurations. This allows even to derive an effective smooth MFA which qualitatively reproduces the SPA behavior [17]. SPA results for other nuclei can be found in $[10,11,16]$. We conclude that the present techniques provide a powerful means to describe finite size effects in small quantum systems at finite temperature. A detailed study of the RPA correlations in this context is in progress.

The authors acknowledge DGICyT, Spain, for grant PB94-0164. RR is a fellow of CICBA and NC of CONICET, of Argentina.

\section{References}

1. Mühlschlegel, B., Scalapino, D.J., Denton, R.: Phys. Rev. B6 1767 (1972)

2. Goodman, A.L.: Phys. Rev. C29 1887 (1984); C37 2162 (1988). Egido, J.L., Ring, P., Iwasaki, S., Mang, H.J.: Phys. Lett. B154 1 (1985)

3. Egido, J.L., Ring, P.: J. of Phys. G19 1 (1993)

4. Gallardo, M., Diebel, M., Døssing, T., Broglia, R.A.: Nucl. Phys. A443 415 (1985). Alhassid, Y., Bush, B., Levit, S.: Phys. Rev. Lett. 611926 (1988). Ormand, W., Bortignon P., Broglia, R.A.: Phys. Rev. Lett. 77 607 (1996)

5. Martin, V., Egido, J.L.: Phys. Rev. C51 3084 (1995)

6. Arve, P., Bertsch, G.F., Lauritzen, B., Puddu, G.: Ann. of Phys. (N.Y.) 183309 (1988). Lauritzen, B., Arve, P., Bertsch, G.F.: Phys. Rev. Lett. 612835 (1988)

7. Alhassid, Y., Zingman, J.: Phys. Rev. C30 684 (1984). Alhassid, Y., Bush, B.: Nucl. Phys. A549 43 (1992); Nucl. Phys. A565 399 (1993)

8. Puddu, G., Bortignon, P.F., Broglia, R.A.: Ann. of Phys. (N.Y.) 206 409 (1991)

9. Lauritzen, B., Puddu, G., Broglia, R.A., Bortignon, P.F.: Phys. Lett. B246 329 (1990); Puddu, G.: Phys. Rev. B45 9882 (1992)

10. Rossignoli, R., Ansari, A., Ring, P.: Phys. Rev. Lett. 709 (1993); Rossignoli, R., Ring, P., Dang, N.: Phys. Lett. B297 9 (1992); Rossignoli, R., Ring, P.: Ann. of Phys. (N.Y.) 235350 (1994)

11. Rossignoli, R., Canosa, N., Egido, J.L.: Nucl. Phys. A605 1 (1996); Nucl. Phys. A607 250 (1996)

12. Hubbard, J.: Phys. Rev. Lett. 377 (1959). Stratonovich, R.L.: Dokl. Akad. Nauk. SSSR 1151097 (1957) (trans. Soviet Phys. Dokl. 2458 (1958)

13. Rossignoli R., Canosa, N.: Phys. Lett. B (1997) (in press)

14. Baranger, M., Kumar, K.: Nucl. Phys. A110 490 (1968); ibid 529 (1968)

15. Alhassid, Y., Bertsch, G., Dean, D., Koonin, S.E.: Phys. Rev. Lett. 77 1444 (1996)

16. Rossignoli, R.: Phys. Rev. C54 1230 (1996)

17. Rossignoli, R., Canosa, N., Ring, P.: Nucl. Phys. A591 15 (1995); Phys. Rev. Lett. 724070 (1994) 